The Maxwell Stability Criterion in Pseudo-Energy Models of Kink Banding

\author{
G.W. Hunt, M.A. Peletier, M. Ahmer Wadee
}

Modelling, Analysis and Simulation (MAS)

MAS-R9911 May 31, 1999 
Report MAS-R9911

ISSN 1386-3703

CWI

P.O. Box 94079

1090 GB Amsterdam

The Netherlands

$\mathrm{CWI}$ is the National Research Institute for Mathematics and Computer Science. CWI is part of the Stichting Mathematisch Centrum (SMC), the Dutch foundation for promotion of mathematics and computer science and their applications.

SMC is sponsored by the Netherlands Organization for Scientific Research (NWO). CWI is a member of ERCIM, the European Research Consortium for Informatics and Mathematics.

Copyright @ Stichting Mathematisch Centrum P.O. Box 94079, 1090 GB Amsterdam (NL) Kruislaan 413, 1098 SJ Amsterdam (NL) Telephone +3120 5929333 Telefax +31205924199 


\title{
The Maxwell Stability Criterion in Pseudo-Energy Models of Kink Banding
}

\author{
Giles W. Hunt \\ Department of Mechanical Engineering, University of Bath, United Kingdom \\ Mark A. Peletier \\ CWI, P.O. Box 94079, 1090 GB Amsterdam, The Netherlands \\ M. Ahmer Wadee \\ Department of Mechanical Engineering, University of Bath, United Kingdom
}

\begin{abstract}
Kink banding, common to many structures in nature and engineering, has several distinctive features-notably highly nonlinear snap-back instability leading to localization and sequential lock-up. The proposed friction model, although simplified, introduces these defining characteristics without obscuring them by including other effects of lesser immediate significance. In the absence of small imperfections or disturbances, linearized theory suggests that in its pre-kinked configuration the system never goes unstable. However, under sufficient applied end-displacement it is shown to be in a state of extreme metastability, such that micro-disturbances would trigger the nonlinear response. To overcome this problem we adopt an energy approach based on a global rather than a local stability criterion. When applied to imperfect systems with small initial misalignments, the critical displacement thus defined shows little of the sensitivity expected from other stability criteria, and provides a useful lower bound on the expected critical displacement and associated load.

We offer the frictional model not as a complete description, but as a prototype to which other components can be added. Thus it provides information on the triggering, localization and lock-up processes, for example, but not on the choice of kink band width which is fixed a priori. Suggestions are provided throughout of extra energy contributions which will extend the model's capability.
\end{abstract}

1991 Mathematics Subject Classification: 73K20, 73G99, 73N20, 73Q05, 73T05, 73V25

Keywords and Phrases: kink banding, layered structures, multilayer folding, geometric nonlinearities, large deflections, instability, Maxwell criterion, localization

Note: This work was partially carried out under CWI Theme 'Modelling, Analysis, and Simulation'

\section{KINK BANDS IN LAYERED STRUCTURES}

The phenomenon of kink banding is known throughout the engineering and geophysical sciences. Associated with layered structures compressed in a layer-parallel direction, it arises for example in stratified geological systems under tectonic compression (Price and Cosgrove 1990), on a micro-scale in wood and fibre composites (Reid and Peng 1997, Fleck 1997), and internally in wound ropes (Hobbs et al. 1995). A number of technical difficulties appear endemic to the modelling of such systems. Continuum formulations based on anisotropic elasticity suffer from a change in character, from elliptic to hyperbolic; 
consequently projected results tend to be unrealistic (Hobbs et al. 1976, p. 207). Even when Cosserat (couple-stress) continua are used to overcome such problems, the large rotations require a specialized, geometrically nonlinear treatment (Fleck et al. 1995, Fleck 1997, Adhikary and Dyskin 1997, Adhikary and Dyskin 1998, Adhikary et al. 1999). Even for the simplest mechanical models, perfect systems tend to exhibit infinite critical loads, with the corollary of extreme sensitivity to small disturbances or geometric misalignments.

We shall concentrate here on the geological manifestation of kink banding, which when seen from a structural mechanics perspective has a number of distinctive features. Simple experiments on stacks of paper (see Figs. 1 and 2) show an intriguing sequence of phases.
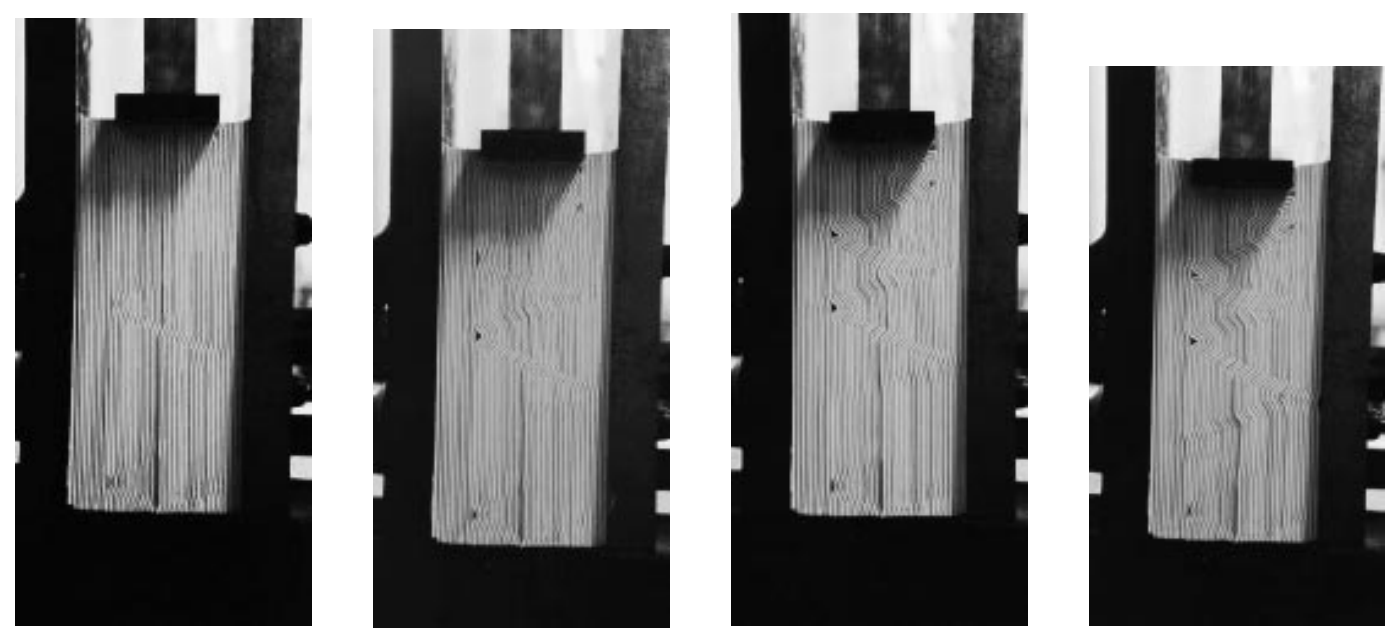

Figure 1: Compression test on layers of paper restrained between rigid faces.

The initial instability appears as a jump phenomenon: it is so unstable that so-called snap-back behaviour is observed where, under conditions of controlled end displacement, the load drops significantly as the system moves suddenly from the flat undeflected state to that of a single kink band involving a large but localized displacement. Yet once the jump has taken place, stability is found in the kinked configuration: the next phase marks a spreading of the bands throughout the specimen, into a periodic pattern with wavelength governed by kink-band angle and specimen width rather than overall length, band width or layer thickness. A final third stage sees transition from a spread of kink bands to overall chevron folding (see Fig. 2), which inevitably involves the migration of fold lines.

The initial, single kink band clearly represents a highly localized form of deformation, where all lateral deflection is concentrated in a narrow band. Kink banding also shows a second form of localization, in the relative tightness of the hinges seen in Fig. 1. Here localization is within the band itself; the deformation is not evenly spread, but concentrated on the boundaries. Experiments suggest that this does not occur to the same degree in all kink bands, and seems to be related to confining pressure: the greater the penalty for the formation of voids between the layers, the sharper the hinges. Plastic yielding is often advanced as the explanation for this localization of strain, but we believe that in the geological framework, plasticity is neither necessary nor sufficient to explain the effect. 

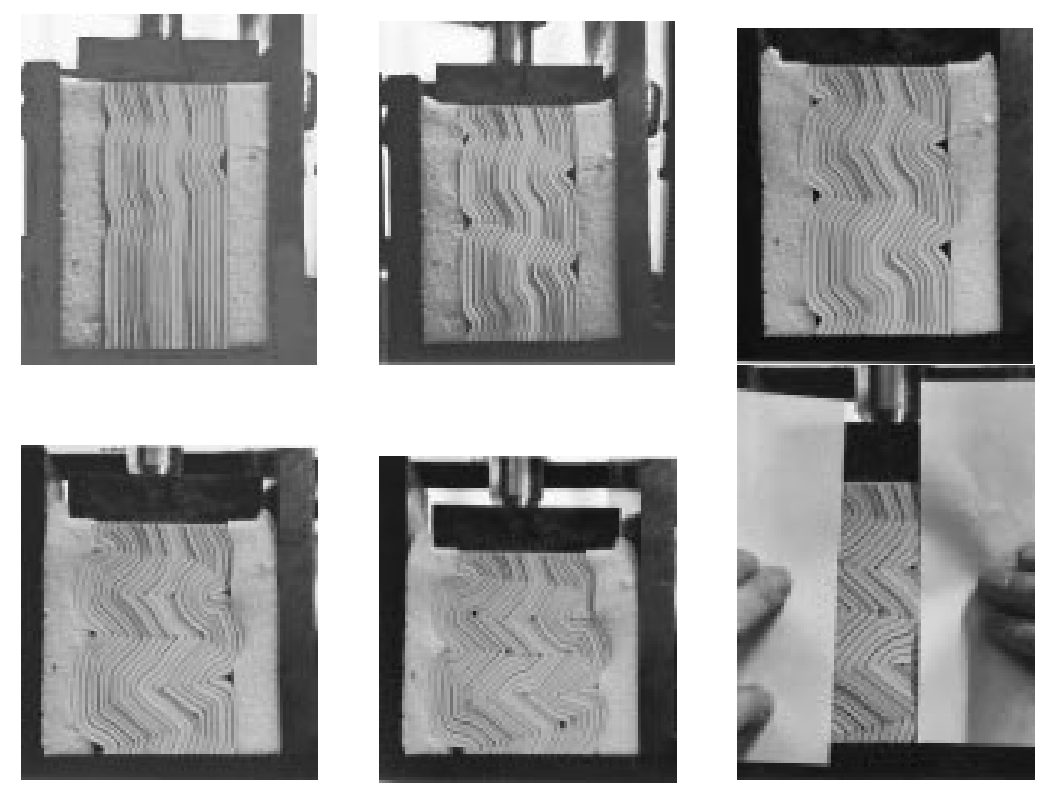

Figure 2: Compression test on layers of paper restrained between flexible faces.

The object of this paper is to reproduce the early stages of the kink-banding process in a simplified model. To reproduce the jump behaviour observed experimentally we give the system an initial layer-parallel stiffness that enables elastic unloading into the kinked region. Inter-layer friction is introduced according to a simple Coulomb law. Because of the large rotations, the model is inherently nonlinear; indeed it is a central tenet that linearized or small-deflection models give results that bear little or no relation to the final outcome. Also, in a move that is more than just simple preference, we adopt an energy or virtual work formulation. This allows us to sidestep the impractical issue of infinite critical loads by adopting the Maxwell criterion of stability - see Zeeman, 1977, p. 53 - arguing that underlying disturbance is such that buckling is triggered when enough strain energy is stored in the initial precompression to overcome internal work against friction: such a system will always seek a global, as opposed to a local, energy minimum. This criterion is known to be useful when localization and locking-up are part of the picture (Hunt et al. 1999), and is seen here to be robust against initial misalignments. The simplified treatment takes kink band width as a priori given, although the addition of bending energy at the hinges should allow this important physical dimension to emerge naturally from the same energy minimization process (see also Budiansky et al. 1998).

The structure of the paper is as follows. In Section 2 we develop in detail the frictional model and explain the locking-up process, in a model in which kinks are orientated orthogonally to the direction of the layers. This is followed by a more complete energy formulation that allows the kink-band angle to form naturally from a minimization process. Sections 3 and 4 are then devoted to an analysis of the model based on the Maxwell criterion, both in perfect form and with imperfections, and in Section 5 we discuss the underlying hypotheses. Finally, an appendix gives detailed support to the argument, assumed at the start of 
the modelling process, that the corners in the kink bands are sharp.

We close this general introduction with a key point in the modelling philosophy. We do not claim that the simplified friction model is necessarily quantitatively accurate. Indeed, as it develops, we will at times identify by footnotes, as well as in the discussion in Section 5, additional energy contributions (work done against overburden pressure in creating voids, for example) that readily could have been included in a more complete development. We note that energy formulations are particularly suited to such 'bolt-on' or modular approaches. Thus the friction model is seen as merely as one of a generic family, which might eventually be combined in a sophisticated model with predictive qualities. For the present, we shall content ourselves with highlighting only the possibility of such extensions.

\section{Prototype Models}

In his book on modelling, Maynard Smith, 1974 distinguishes two types of mathematical models. 'Simulations' are large and complex constructions that endeavour to recreate the system under investigation in as many aspects and as much detail as possible. Because of the complexity the only reasonably possible manipulation of such models is numerical, using computers to perform numerical experiments. Maynard Smith reserves the term 'model' for a different species of mathematical model, which is often - deprecatinglytermed 'toy model'. Where the 'simulation' is geared to provide answers to a broad range of questions, many of which are unknown at the time of conception, a toy model is conceived and constructed to provide a specific type of answer to a specific question. This a priori restriction of purpose provides a much greater freedom in the methods of modelling that are used, and allows for ruthless simplification. The resulting models are invariably much simpler than in the case of the 'simulations', and tend to be more accessible to analysis. The insight gained through this approach can be of prime importance in understanding more complex models and the real-world system itself.

\subsection{Vertical Stacking}

Consider the stack of layers of Fig. 3. Fig. 4 shows a representative two-layer section

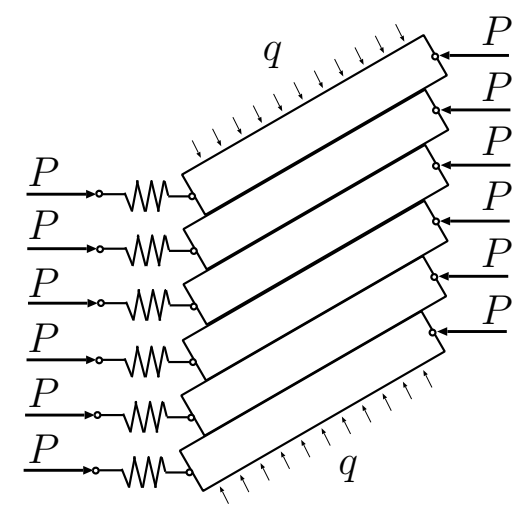

Figure 3: Full stack of blocks, showing external loading and inline springs.

from the middle of this stack. Each layer is of thickness $t$, preset length $b$, and supports 


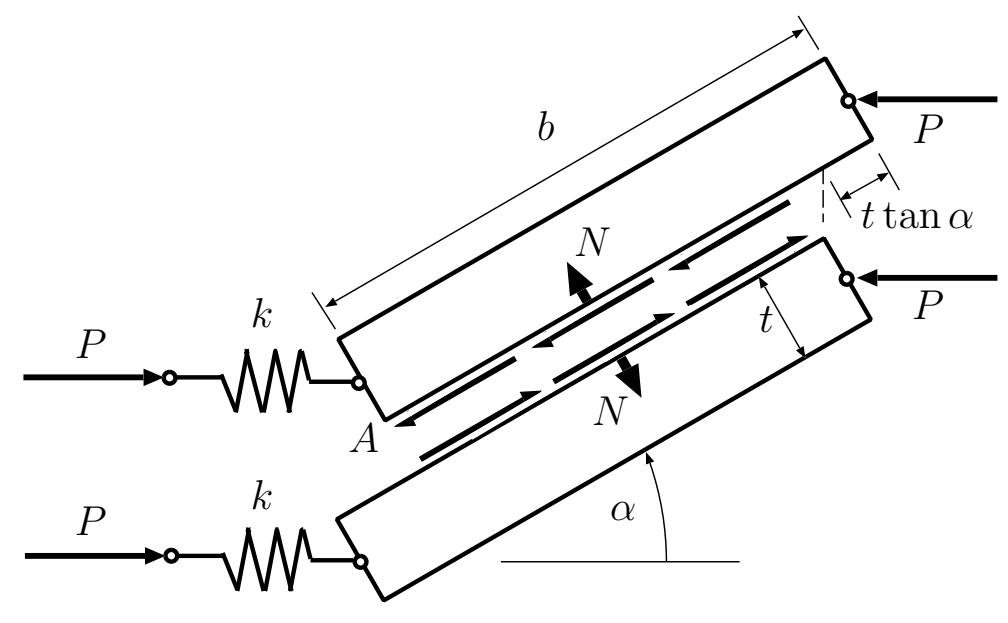

Figure 4: Representative two-layer section

a conservative load $P$. The upper and lower exposed surfaces are subject to a fixed distributed normal load $q$, representing the overburden pressure. Layer-parallel stiffness is represented by inline springs of stiffness $k$. For ease of description the layers are aligned vertically, although in practice they would be inclined; we return to the inclined geometry in Section 2.2. No energy terms are included to represent work done at the sharp hinges or folds, and to compensate, the kink band width $b$ is fixed a priori. (Bending energy in the hinges is expected to be significant in determining the true value of $b$, as seen later.) The present formulation allows us to introduce the nonlinear behaviour and associated Maxwell criterion for instability in an uncluttered way.

The equilibrium equations for this system are derived by minimizing a total potential energy function, constructed by adding separate potentials for each of the components. Although friction is not conservative, we assume that layers slide in the same direction throughout the deformation process; this allows for a pseudo-energy description of the work done by friction.

The interlayer forces are supposed to consist of a normal force $N$ and a friction force $A$, which for simplicity both are taken to be point forces. Assuming a Coulomb friction law, $A$ satisfies $0 \leq A \leq \mu N$, where $\mu$ is the coefficient of friction ${ }^{1}$. We obtain from the vertical equilibrium condition for the top block in Fig. 3,

$$
q b \cos \alpha=N \cos \alpha-A \sin \alpha .
$$

If $A$ is assumed to be always at the critical state $A=\mu N$, we can single out $N$,

$$
N=\frac{q b}{1-\mu \tan \alpha}
$$

\footnotetext{
${ }^{1}$ Note that the friction force $A$ could take other forms, leading to different but related responses. For example, if $A$ is taken constant, the response would have much in common with the plastic shear model of Budiansky et al., 1998.
} 
Note that $N$ is only positive if $\alpha<\operatorname{arccot} \mu$. Using moment equilibrium for a typical middle block,

$$
P=\frac{N t(\tan \alpha+\mu)}{b \sin \alpha}=\frac{q t}{\sin \alpha} \frac{\mu+\tan \alpha}{1-\mu \tan \alpha}
$$

The work done by the friction force is then

$$
\begin{aligned}
W_{F r} & =-\int_{0}^{\alpha} \mu N\left(\alpha^{\prime}\right) d\left(t \tan \alpha^{\prime}\right)=-\int_{0}^{\alpha} \frac{q b \mu}{1-\mu \tan \alpha^{\prime}} d\left(t \tan \alpha^{\prime}\right) \\
& =-\mu q b t \int_{0}^{\alpha} \frac{\sec ^{2} \alpha^{\prime}}{1-\mu \tan \alpha^{\prime}} d \alpha^{\prime}=q b t \ln (1-\mu \tan \alpha),
\end{aligned}
$$

provided $\alpha<\operatorname{arccot} \mu$. Since the layers slide in the same direction throughout the deformation, we can interpret this work as a pseudo-energy,

$$
V_{F r}=-W_{F r} \text {. }
$$

From this energy term we subtract the work done by the point forces $P$ and the distributed force $q$ (Fig. 3):

$$
\begin{aligned}
& W_{P}=P b(1-\cos \alpha) \\
& W_{q}=-\int_{0}^{\alpha} q b \cos \alpha^{\prime} d\left(t / \cos \alpha^{\prime}\right)=-q b t \int_{0}^{\alpha} \tan \alpha^{\prime} d \alpha^{\prime}=q b t \ln \cos \alpha
\end{aligned}
$$

and we add the potential associated with the compression $\delta$ of the springs $k$,

$$
V_{k}=\frac{1}{2} k \delta^{2}-P \delta
$$

This yields the total potential energy

$$
\begin{aligned}
V & =V_{k}+V_{F r}-\left(W_{P}+W_{q}\right) \\
& =\frac{1}{2} k \delta^{2}-q b t \ln (\cos \alpha-\mu \sin \alpha)-P[\delta+b(1-\cos \alpha)] .
\end{aligned}
$$

At this point we replace $P$, a point force, by $p$, a distributed force, which are related by $P=p t$. This makes $p$ commensurate with $q$ and will be more convenient for later models. In addition, we non-dimensionalize the problem by considering $t$ as a typical length scale and $k$ as a measure of force per unit area. Setting

$$
\delta=t \tilde{\delta}, \quad b=t \tilde{b}, \quad q=k \tilde{q}, \quad p=k \tilde{p}
$$

and dropping the tildes immediately, the non-dimensionalized potential energy is

$$
V=\frac{1}{2} \delta^{2}-q b \ln (\cos \alpha-\mu \sin \alpha)-p[\delta+b(1-\cos \alpha)] .
$$


Stationary points of this energy satisfy the two equilibrium equations

$$
\begin{aligned}
& p=\delta \\
& p=\frac{q}{\sin \alpha} \frac{\mu+\tan \alpha}{1-\mu \tan \alpha} .
\end{aligned}
$$

The interesting curve to plot is that of $p$ against $\Delta$, the total end-shortening given by

$$
\Delta=\delta+b(1-\cos \alpha)
$$

By parameterizing in $\alpha$, the variation of $p$ with end-shortening $\Delta$ over a range of different but constant $q$ values is as shown in Fig. 5. Each curve approaches both the flat state $\alpha=0$ and the locked-up state $\alpha=\operatorname{arccot} \mu$ asymptotically as $p \rightarrow \infty$. The curves imply among other things the absence of critical bifurcation loads for $q \neq 0$ over the full range of finite $p$.

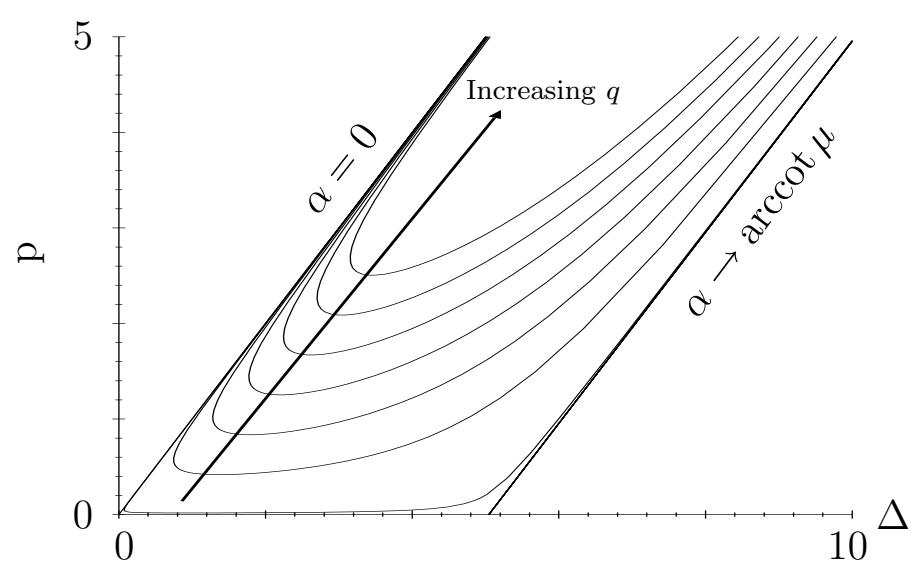

Figure 5: Equilibrium solutions on a plot of load $p$ against total end-shortening $\Delta$, for varying $q$ with constants $\mu=0.57$ and $b=10$.

\subsection{Inclined Geometry}

Actual kink bands do not form orthogonal to the layering. A simple explanation is that the increase in height $(t \mapsto t / \cos \alpha)$ occupied by a layer in the vertical stack forces the adjoining layers apart; the voids thus created represent significant work against the overburden pressure. A more realistic mode of deformation is shown in the stylized geometry of Fig. 6 . As the angle $\alpha$ increases, the angle $\beta$ of the band also increases, and provided that $\alpha$ remains equal to $2 \beta$ throughout the deformation the layer distance is the same in the kink band as in the unbuckled layers.

The inclined geometry leads to two changes in the model outlined in the previous sections. First, the distance that two layers slide with respect to each other is now $t \tan (\alpha-\beta)=$ 

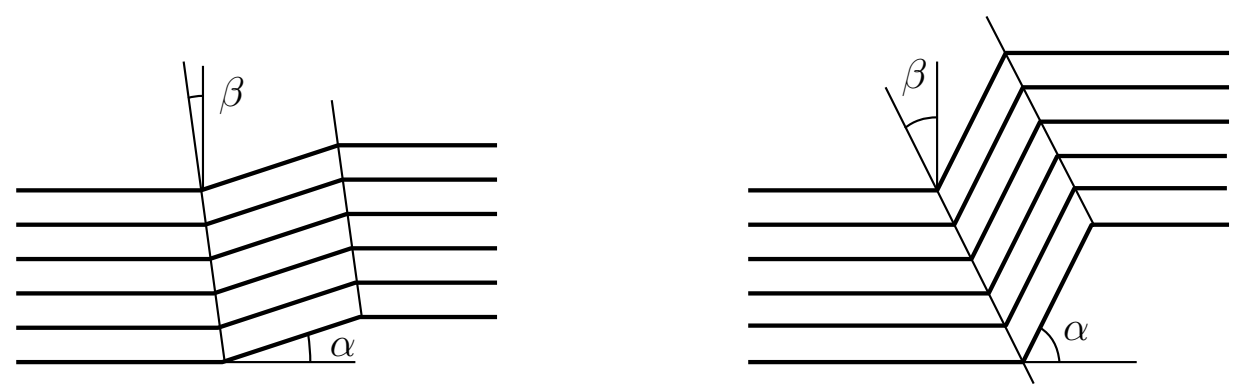

Figure 6: If the distance between layers is to remain constant, the kink band angle $\beta$ must vary according to $\alpha=2 \beta$.

$t \tan \alpha / 2$. For this geometry the friction 'energy' becomes, in non-dimensionalized form,

$$
V_{F r}=-W_{F r}=\int_{0}^{\alpha} \mu N\left(\alpha^{\prime}\right) d\left(\tan \alpha^{\prime} / 2\right)=\frac{\mu q b}{2} \int_{0}^{\alpha} \frac{\sec ^{2}\left(\alpha^{\prime} / 2\right) d \alpha^{\prime}}{\left(1-\mu \tan \alpha^{\prime}\right)} .
$$

Although this integral has an explicit solution, we leave it as it stands for brevity. Secondly, the work done by $p$ and against $q$ needs to be recalculated. The total work done by these external forces was determined as $p b(1-\cos \alpha)+q b \log \cos \alpha$ in the original, vertical, stack of layers. For convenience we split $p$ into two parts, $p=p_{1}+q$, such that $p_{1}$ is the layer-parallel applied load that is additional to $q$. Since $\alpha=2 \beta$ the deformation is volume-conserving ${ }^{2}$, and thus $q$ does no work (see Fig. 7). The total work done by $p$ and $q$ (per single layer, as before, and in non-dimensionalized form) is therefore equal to $W_{p}+W_{q}=p_{1} b(1-\cos \alpha)=(p-q) b(1-\cos \alpha)$. In the tilting geometry the energy thus becomes,

$$
V=\frac{1}{2} \delta^{2}-p \delta+\frac{\mu q b}{2} \int_{0}^{\alpha} \frac{\sec ^{2}\left(\alpha^{\prime} / 2\right) d \alpha^{\prime}}{\left(1-\mu \tan \alpha^{\prime}\right)}-(p-q) b(1-\cos \alpha)
$$

and the equilibrium equations are

$$
\begin{aligned}
p & =\delta \\
\frac{p}{q} & =1+\frac{\mu}{\sin \alpha(1+\cos \alpha)(1-\mu \tan \alpha)} .
\end{aligned}
$$

Fig. 8 shows the equilibrium curves of both the vertical and the inclined kink bands. Because the layers slide less in the inclined geometry $(\tan \alpha / 2$ instead of $\tan \alpha)$ the friction

\footnotetext{
${ }^{2}$ Note that if the condition $\alpha=2 \beta$ is relaxed, and $\beta$ is allowed to take values larger than $\alpha / 2$, the volume is not conserved - the layers inside the kink band separate (dilatation) and this results in the frictional force, associated with the sliding of the layers, being released. This dilatation introduces an extra component of work done against overburden pressure $q$ because voids are penalized. Thus, during a deformation process where dilatation is significant, there is a complex interplay between the release of friction and the work done against $q$. When the deformed region returns to its original volume $(\alpha=2 \beta)$, contact reintroduces frictional forces and the response returns to that of the above model. We intend to cover the dilatation possibility in future work.
} 


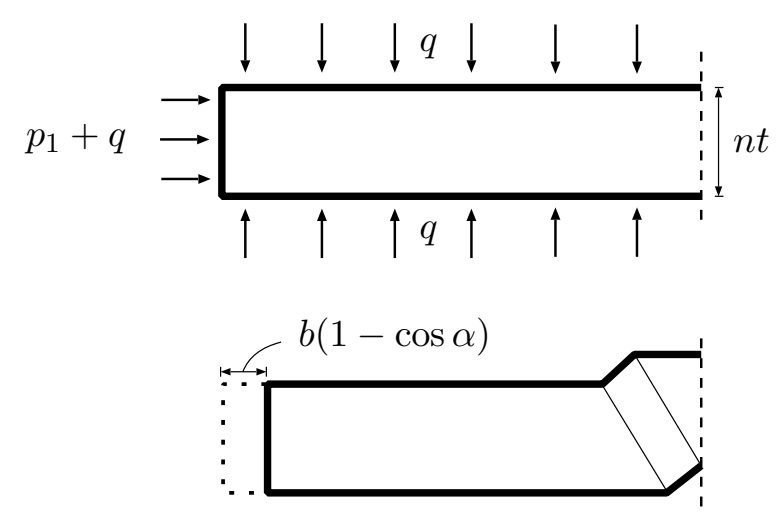

Figure 7: Work done by the pressures $p$ and $q$ during the inclined-geometry deformation. The stack contains $n$ layers, and the dashed vertical line denotes an axis of symmetry.

energy is less, leading to a smaller total strain energy. This is a different energetic advantage from the reduction in voids between adjoining layers, that determines the geometry of straight limbs and sharp corners.

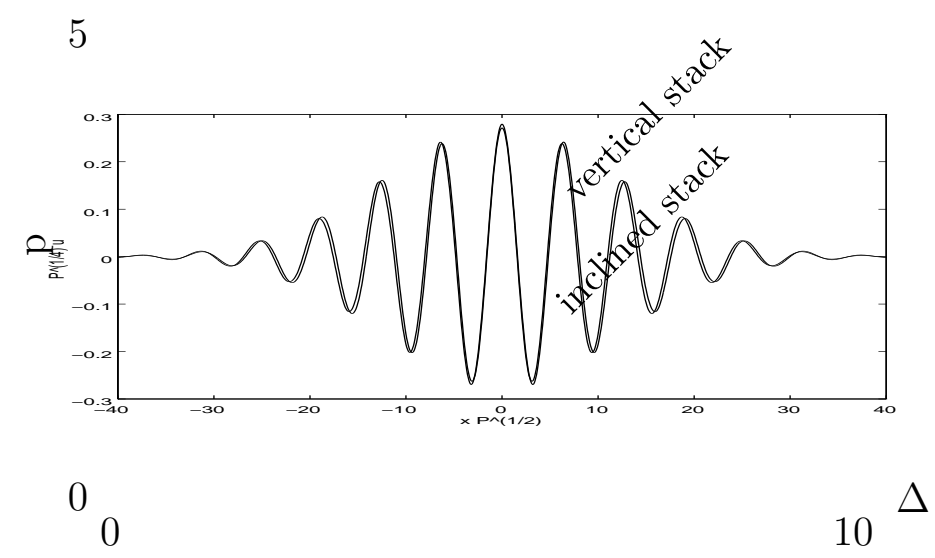

Figure 8: Equilibrium curves for the straight and for the inclined geometry. Here $q=0.5$, $\mu=0.57$, and $b=10$.

\section{Maxwell Stability Criterion}

Starting from the undeflected state, varying either load $p$ or end-displacement $\Delta$ would cause the system to move up the straight line in Fig. 5. As all equilibria on this path are local energy minima, according to the standard delay ${ }^{3}$ stability convention the critical load is infinite. However, high loads imply an extreme state of metastability, as the slightest disturbance would trigger the jump response. In this situation the classical approach based on linear eigenvalue analysis is of little value. An inherently nonlinear method is required, based on large deformations and geared to stability in the presence of perturbations. As

\footnotetext{
${ }^{3}$ This convention can be described as 'stay in an equilibrium state as long as it is a local energy minimum; move when this fails.'
} 
such a system may respond differently depending on whether the load or end-displacement is controlled, the loading conditions first need to be carefully specified.

Here we assume rigid loading conditions such that end-shortening $\Delta$ is the controlled parameter (Thompson and Hunt 1973). To cover the problem of infinite critical loads we adopt the so-called Maxwell stability criterion (Zeeman, 1977, p. 53; see also Biot, 1965, p. 204 for an early mention in the geological context) where stability rests only with the global, as opposed to any local, minimum of total potential energy. The Maxwell law is commonly used for thermodynamical instability, for instance in modelling phase transitions where underlying disturbances and statistical fluctuations ensure that the system shakes out of local minima and seeks a global energy minimum. The same criterion has recently been applied to a number of structural problems with the destabilizing/restabilizing (localizing/locking-up) characteristics as found here (Hunt et al. 1999).

Consider for the moment a typical load/end-shortening response graph for an elastic structure which twice bends back on itself, as shown schematically in Fig. 9. If $\Delta$ is the

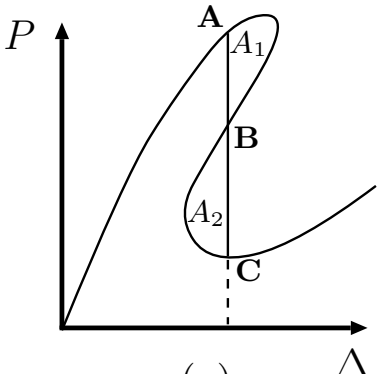

$(a)$

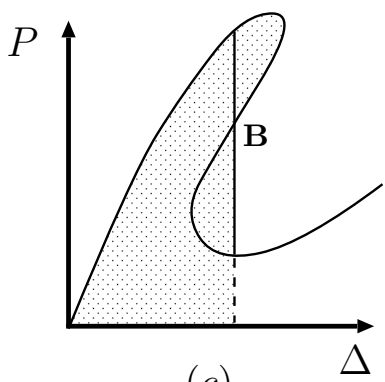

$(c)$

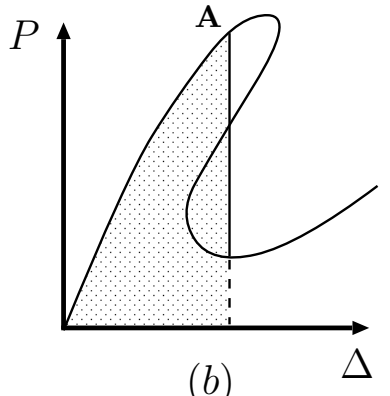

$(b)$

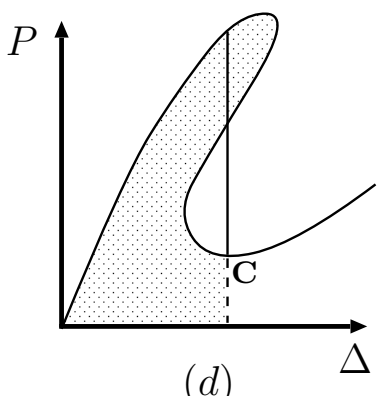

$(d)$

Figure 9: Strain energy under controlled end-shortening $\Delta$. (a) Three possible equilibrium states. (b) Strain energy at A. (c) Strain energy at B. (d) Strain energy at C.

controlled parameter, the area under the graph represents the strain energy stored. Over a certain range of $\Delta$ the response is triple-valued. Of the three possible candidates for equilibrium, $\mathbf{A}$ and $\mathbf{C}$ are local energy minima and $\mathbf{B}$ is a local maximum.

Stored strain energies at $\mathbf{A}, \mathbf{B}$ and $\mathbf{C}$ are as shown; area $A_{1}$ thus represents the energy hump to be overcome in moving from $\mathbf{A}$ to $\mathbf{B}$, while area $A_{2}$ is energy that is available for release in moving from $\mathbf{B}$ to $\mathbf{C}$. For the 'delay' stability convention the system may remain at either $\mathbf{A}$ or $\mathbf{C}$, while under the Maxwell criterion, stability rests only with state of lower 
energy. The Maxwell displacement $\Delta_{\mathrm{M}}$ is the value of end-shortening where the minimum swaps from $\mathbf{A}$ to $\mathbf{C}$, and hence $A_{1}=A_{2}$.

The Maxwell criterion seems particularly apt when applied to a system with a response like that of Fig. 5. $A_{1}$ is now the work done against friction; it continues to $p=\infty$ yet is very thin - more of an energy spike than energy hump - and by nature would be extremely sensitive to imperfections. $A_{2}$ is the energy available from the pre-compression, and is of a generally more robust shape. The net effect is that a slight increase in $\Delta$ from the Maxwell position will change $A_{1}$ only a little, but enlarge $A_{2}$ considerably. Thus not only is the spike easily eroded by imperfections, but the stored energy available for release is significantly enhanced by only a small change in $\Delta$. The Maxwell displacement thus provides a robust lower bound to the actual critical displacement for kink banding.

We will now calculate the value of $\Delta_{M}$ and the associated value $\alpha_{M}$ for both the vertical and the inclined stack. Fig. 10 shows the situation in caricature. Note that $p \rightarrow \infty$ as

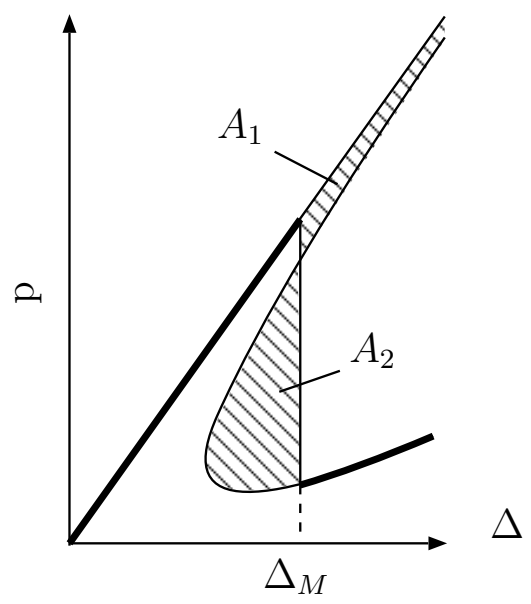

Figure 10: Equilibrium solutions on a plot of load $p$ against total end-shortening $\Delta$.

$\alpha \rightarrow 0$ on the upper, and similarly $p \rightarrow \infty$ as $\alpha \rightarrow \operatorname{arccot} \mu$ on the lower, branch of the curve in Fig. 5. On integrating from $\alpha=0$ to the value $\alpha=\alpha_{M}$, the area of interest, shaded in Fig. 9, is given by

$$
\text { Area }=\int_{\alpha=0}^{\alpha=\alpha_{M}}(p-\Delta) \mathrm{d} \Delta .
$$

\subsection{Vertical stack}

Differentiation of (5) gives $d \Delta=d \delta+b \sin \alpha d \alpha$, and after substituting the equilibrium equations (3-4) this integral can be written,

$$
\text { Area }=-\left.\frac{1}{2} \Delta^{2}\right|_{0} ^{\alpha_{M}}+\left.\frac{1}{2} \delta^{2}\right|_{0} ^{\alpha_{M}}+\left.q b \ln (\cos \alpha-\mu \sin \alpha)\right|_{0} ^{\alpha_{M}} .
$$


If it is zero the Maxwell condition is satisfied for a jump from the upper straight line representing the undeflected state, to the lower branch of the curve. Substituting for $\Delta, \delta$, and $p$ in terms of $\alpha$ then leaves the equation

$$
\begin{aligned}
& \ln \left(\cos \alpha_{M}-\mu \sin \alpha_{M}\right)-\frac{\left(1-\cos \alpha_{M}\right)\left(\sin \alpha_{M}+\mu \cos \alpha_{M}\right)}{\sin \alpha_{M}\left(\cos \alpha_{M}-\mu \sin \alpha_{M}\right)} \\
& \quad=\frac{b}{2 q}\left(1-\cos \alpha_{M}\right)^{2},
\end{aligned}
$$

from which the particular Maxwell displacement $\Delta_{M}$ can be found, where energy levels in pre-buckled $(\alpha=0)$ and post-buckled $\left(\alpha=\alpha_{M}\right)$ states match. Fig. 11 shows the values of $\Delta_{M}$ for two different values of $q$.

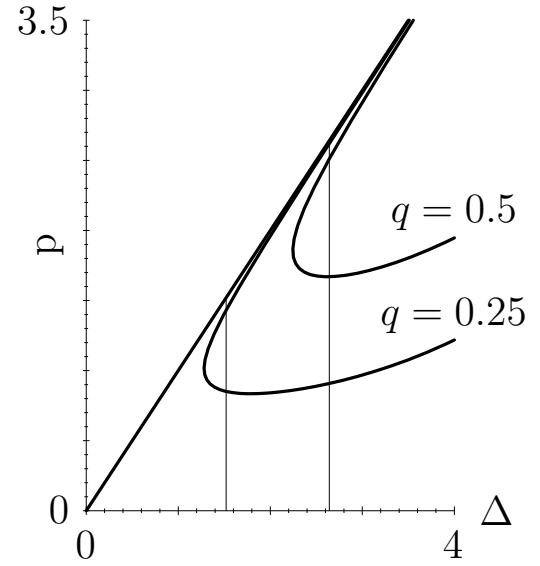

(a) Vertical stack

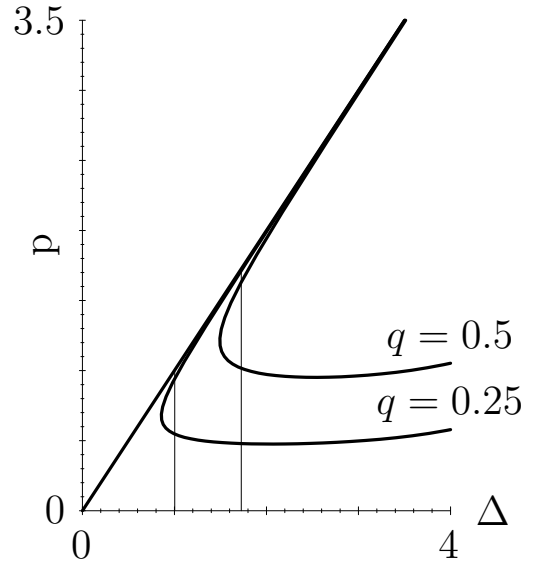

(b) Inclined stack

Figure 11: Maxwell displacements $\Delta_{M}$.

\subsection{Inclined stack}

Similarly, for the inclined stack, Equation (8) can be written as

$$
\text { Area }=-\left.\frac{1}{2} \Delta^{2}\right|_{0} ^{\alpha_{M}}+\left.\frac{1}{2} \delta^{2}\right|_{0} ^{\alpha_{M}}+q b\left[\int_{0}^{\alpha_{M}} \frac{\mu \sec ^{2}(\alpha / 2) d \alpha}{2(1-\mu \tan \alpha)}+\left.(1-\cos \alpha)\right|_{0} ^{\alpha_{M}}\right]
$$

and substituting for $\delta, \Delta$, and $p$, gives the following equation at the Maxwell displacement $\left(\alpha=\alpha_{M}\right)$ :

$$
\begin{aligned}
\int_{0}^{\alpha_{M}} & \frac{\mu \sec ^{2}(\alpha / 2) d \alpha}{2(1-\mu \tan \alpha)}-\frac{1-\cos \alpha_{M}}{\sin \alpha_{M}\left(1+\cos \alpha_{M}\right)\left(1-\mu \tan \alpha_{M}\right)} \\
& =\frac{b}{2 q}\left(1-\cos \alpha_{M}\right)^{2}
\end{aligned}
$$




\begin{tabular}{|c|c|c|c|}
\hline$\alpha_{0}$ & $p_{\text {peak }}$ & $\Delta_{M}$ & $p_{M}$ \\
\hline 0 & $\infty$ & 1.726 & 1.726 \\
0.05 & 3.437 & 1.637 & 1.625 \\
0.1 & 2.018 & 1.570 & 1.520 \\
0.2 & 1.319 & 1.499 & 1.300 \\
\hline
\end{tabular}

Table 1: Comparison of imperfect peak loads $p_{\text {peak }}$ against Maxwell displacements $\Delta_{M}$ and corresponding loads $p_{M}$.

\section{IMPACT OF IMPERFECTIONS}

In reality, the infinite critical load of the perfect system is not expected to survive the introduction of misalignments or other small imperfections. As we see below the infinite spike is lost, and we witness the appearance of a 'peak load' at which the unbuckled state loses local stability. The level of this peak load depends strongly on the magnitude of the imperfections; in fact, in the limit of vanishing imperfections the peak load must rejoin the perfect-system critical load at infinity. As a result of this property a large body of research has developed into the impact of imperfections on the peak load. (For an overview in the field of fibre-reinforced composites, see Fleck 1997).

Part of our interest in the Maxwell criterion is motivated by the fact that the critical displacement given by this criterion, and the corresponding load at this displacement, are much less sensitive to the size of the imperfections than is the peak load. In the example that we study below this becomes clear.

\subsection{Initial misalignment}

As an example of an imperfection we commence loading at a small initial misalignment ${ }^{4}$, i.e. we start with $\alpha=\alpha_{0}>0$. Fig. 12 shows the load-deflection diagram for three different non-zero values of $\alpha_{0}$. Note that the definition of $\Delta$ is unchanged, so that $\alpha_{0}>0$ implies $\Delta_{0}>0$, and that the initial misalignment does not then change the position or shape of the post-buckled equilibrium curve. Observe the wide variation in the peak load with different values of $\alpha_{0}$, and the corresponding narrow variation of Maxwell displacement $\Delta_{M}$, and associated load $p_{M}$, as shown in Table 1 . We conclude that the imperfection removes only a small amount of energy from the total energy hump that needs to be overcome by outside disturbances.

\section{Comments on the MOdelling}

From a model as simplified as the one above one should not necessarily expect realistic quantitative results. In the spirit of Maynard Smith, 1974 it is constructed with two purposes in mind:

\footnotetext{
${ }^{4}$ In the case presented the only difference in the perfect and imperfect responses is in the unbuckled path. In accordance with nonlinear bifurcation theory (Golubitsky and Schaeffer 1984), there could be other types of imperfection which affect both the unbuckled and the post-buckled state, and create a smooth transition between the two. With such an imperfection the system would also have a peak load, and respond such that the imperfect path is asymptotic to the perfect system for large rotations.
} 


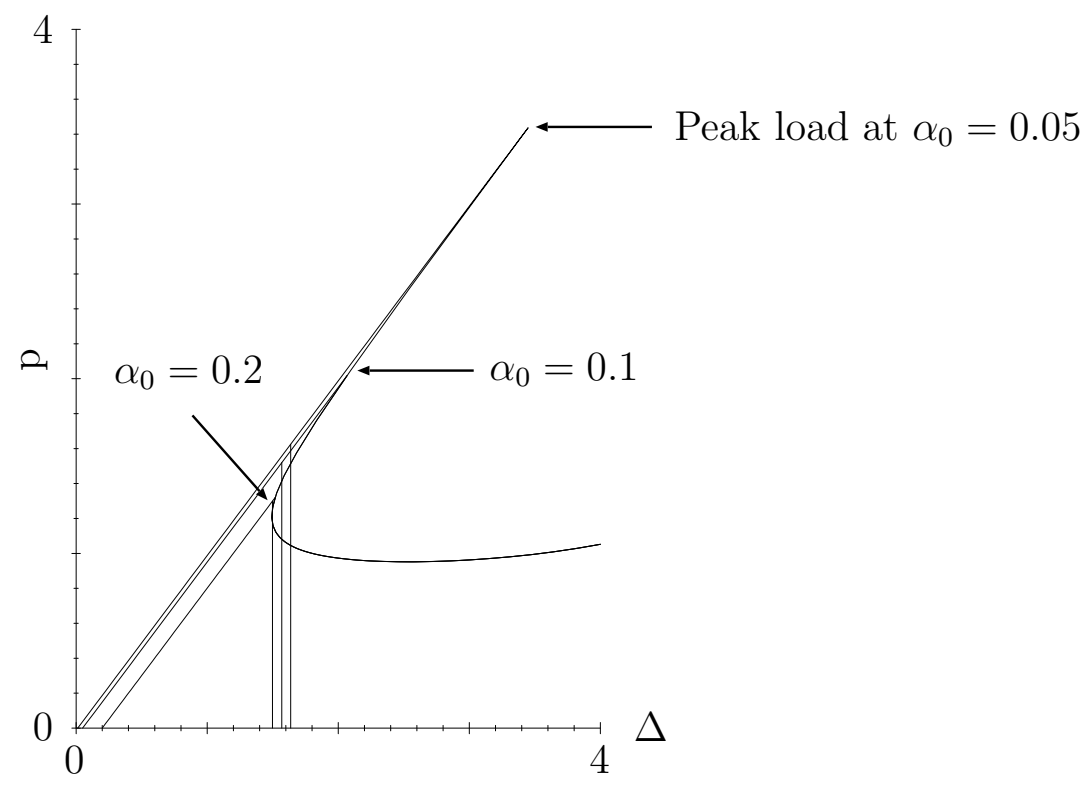

Figure 12: Variations of peak load and Maxwell displacement with initial misalignments. Here $\mu=0.57$ and $q=0.5$.

1. To demonstrate application of the Maxwell criterion to imperfection-sensitive snapback phenomena such as kink banding;

2. To help select the most significant modelling characteristics of the kink-band phenomenon from amongst all possible competing alternatives.

\subsection{The Maxwell criterion}

The Maxwell criterion is normally applied where load rather than displacement is the controlled parameter (although we note its appearance in Budiansky et al., 1998 in the related context of the broadening of kink bands). When displacement is controlled and disturbances are rife - folding of geological strata being an excellent example - the Maxwell criterion should provide a realistic and robust lower bound on the actual end-shortening displacement at the first instability. It is however necessary to draw a distinction between the roles of external disturbance and inherent imperfections. While imperfections are local destabilizing factors, in the elastic setting of the pre-compression external disturbances are more likely to appear on the scale of the overall structural dimension. Their effect might therefore be expected to depend on sample length. Add to this the fact that long samples have more severe snap-back characteristics than short ones, and we can speculate that Maxwell predictions would be most successful when structures are long and failures are local.

To illustrate this, let us assess the strength of a layered structure as studied in this paper. For the moment we assume that we know the structure in detail, including any initial misalignments, but that it is exposed to unknown external disturbances. Suppose it moves, under controlled shortening, along the unbuckled path, until a buckle is triggered. 
It is reasonable that the peak load is an upper bound of the strength, since disturbances will cause the system to buckle before the peak load is reached. On the other hand, the Maxwell load is a reasonable lower bound for the strength, since below this value a positive energy input from the disturbance is necessary to bring the system to the deflected state.

Moving to a more realistic setting where the magnitude of the misalignment is not known, when peak loads and Maxwell loads are compared for different misalignments, we observe that the variation is much larger in the former than in the latter. From a practical point of view the Maxwell load could thus provide a more robust starting point for estimations of strength than the peak load.

\subsection{The relative importance of assumptions for kink banding}

The model discussed here contains three basic ingredients: the inline springs, friction between the layers, and the external forces $p$ and $q$. This is a matter of choice: we have left out (other) elastic, viscous, and plastic properties, the influence of pore fluid, and many other factors. In addition we assume a highly simplified geometry with a constraint that, if interpreted strictly, implies that hinge lines migrate. (Despite the high degree of simplification in the geometry, one element that is left unchanged is the strongly geometrically non-linear character of the folding.) All these assumptions are clearly incorrect for actual materials, but they allow us to make one clear statement:

The essence of kink-band formation is captured by layer-parallel stiffness, interlayer friction, and overburden pressure.

We discuss this claim in detail below.

1. Layer-parallel stiffness. Both experimental and numerical evidence indicate that the formation of kink bands is a jump phenomenon, in which previously stored energy is released. The layer-parallel stiffness provides this storage.

2. Interlayer friction. As the layers rotate, the lever arm of the moment associated with $p$ increases. For the folds to lock up, a counteracting force is necessary. Friction fulfils this necessity; there is - reasonably - only one alternative in this role, in the form of a deflection-dependent overburden pressure $q$. However, in the presence of $q$ we would still expect friction to dominate the lock-up state and so it is sensible, at least initially, to involve only the latter.

3. Overburden pressure. The pressure $q$ does however play an important background role. First, a strong overburden pressure in relation to the bending energy of the layers yields folds with straight limbs and tight hinges (see the Appendix). Without overburden pressure, folds would tend to loosen and become rounded, creating voids between the layers. Also, any work done during hinge migration will be small by comparison. Secondly, the overburden pressure acts as a measure for $p$, in the sense that in equation (7) $p$ appears in the combination $p / q$. For the behaviour of the stack - as opposed to the inline springs - it is therefore not the magnitude of $p$ that is important, but its value relative to $q$.

While we have shown that the combination of these three elements provides a convincing structure, one might still wonder whether adding on additional elements or assumptions, 
or relaxing some of the present hypotheses, would in some way improve the model. We mention a few here, accompanied by some remarks.

1. Bending energy and plastic hinges. Although the tight-hinge geometry is based on the assumption that the bending energy is dwarfed by the overburden pressure, one might still imagine adding a bending energy (either of elastic or of plastic type) to the potential $V$ in (2) or (6). To take the second model as an example, if we add to $V$ the term $K \alpha^{2} / 2$ - for an elastic hinge - this leads to the equilibrium equation

$$
\frac{p}{q}=1+\frac{\mu}{\sin \alpha(1+\cos \alpha)(1-\mu \tan \alpha)}+\frac{K}{b t} \frac{\alpha}{\sin \alpha} .
$$

Although the numerical value of $p$ is slightly altered by this addition, the main characteristic of the equilibrium curve $p$ becomes large as $\alpha \rightarrow 0$ and as $\alpha \rightarrow \operatorname{arccot} \mu$-remains completely unaltered. Consequently the adding of such a term has only a numerical influence on the model, without changing its character. It may however play an important role in determining the value of $b$.

The same is true for an additional term of the form $K \alpha$, representing the pseudo-energy associated with a plastic hinge. This results in the equilibrium equation

$$
\frac{p}{q}=1+\frac{\mu}{\sin \alpha(1+\cos \alpha)(1-\mu \tan \alpha)}+\frac{K}{b t \sin \alpha} .
$$

The new term is now singular at $\alpha=0$, but again the overall character of the equilibrium curves has not changed.

2. Embedding energy. In a similar vein, elastic or plastic embedding energy could be introduced as an alternative to overburden pressure. Whereas the constant pressure of a plastic foundation could be seen as similar in nature to $q$, the elastic setting would generate terms of a different kind. Questions of whether Winkler or other foundation assumptions would be appropriate are also relevant, but are left unaddressed at this stage.

3. Hinge migration. In folded geological structures tight hinges can develop secondary deformation structures (e.g. tension gashes in the outer arc). If hinges slowly migrate before reaching their final position, one would expect to find these secondary structures not only in the final position but also along the migration path. The lack of such features in observed strata is sometimes mentioned as an objection to hinge migration.

For the model considered in this paper the paradoxical situation arises that although in the derivation of the model the hinges migrate - and migrate over substantial distances - in fact they never actually do. This is related to the concept of the Maxwell jump criterion, and is illustrated in Fig. 10. The thick line and the thin line together represent all equilibrium configurations. Based on these configurations, the Maxwell displacement $\Delta_{M}$ is determined. In an actual system, represented by this model, we expect that under controlled end-shortening $\Delta$ the system follows the thick line. For instance, starting from zero displacement, we move up the straight line; at the Maxwell displacement the system jumps to the point on the thick curve below and continues up this curve. In this jump, which in practice happens very quickly, the configuration has changed from $\alpha=\beta=0$, i.e. no 
buckling, to $\alpha=\alpha_{M}$, with $\beta=\beta_{M}=\alpha_{M} / 2$. Therefore the conceptual hinge migration, with $\beta$ moving gradually from 0 to $\beta_{M}$-corresponding to all the thin-lined parts of the graph - in fact happens in a jump. The hinge does not migrate, but appears instantly at the Maxwell value.

\section{Concluding Remarks}

The phenomenon of kink banding is seen in many physical systems. In this paper we have presented a model for stratified geological systems, based on pseudo-energy, accounting for the interlayer friction and the presence of overburden pressure. The pseudo-energy approach conveniently allows the implementation of the Maxwell stability criterion. This has been of fundamental importance in the qualitative insight it gives into the structural behaviour and in its potential to gain quantitative information; in the perfect case the physical model exhibits an infinite critical load, but the Maxwell criterion gives a finite value for the system to jump from an unbuckled to buckled shape at equal energies. Two prototype models have been presented: a vertically aligned stack of layers - simple to model but physically unrealistic; and an inclined stack - more complex but physically more realistic. Numerical comparisons have been discussed briefly.

Initial imperfections have also been considered, which introduced a finite peak load and reflected an upper bound on the behaviour. Applying the Maxwell criterion to the imperfect model gives a lower bound to the load at which the system loses stability. This lower bound seems to be much less sensitive to the magnitude of the initial imperfection than the peak load.

The modelling has been done in a modular way so that, as far as possible, extra effects can be added later. Potential additions have been identified and discussed qualitatively through the paper. In future work we intend to use these qualitative discussions and add to the current modelling framework such that the kink banding models are more robustly defined.

\section{Acknowledgements}

The authors would like to thank Dr Ray Lawther from the University of New South Wales, Australia, for his invaluable comments. This work was supported by the EPSRC, through grant GR/L17177 of the Applied Nonlinear Mathematics Initiative. 


\section{References}

Adhikary, D. P., Dyskin, A. V., 1997. A cosserat continuum for layered materials, Computers and Geosciences 20, 15-45.

Adhikary, D. P., Dyskin, A. V., 1998. A continuum model of layered rock masses with nonassociative joint plasticity, International Journal of Numerical Analysis and Methods in Geomechanics 22, 245-261.

Adhikary, D. P., Mühlhaus, H.-B., Dyskin, A. V., 1999. Modelling the large deformations in stratified media - the Cosserat continuum approach, International Journal of Cohesive and Frictional Materials. To appear.

Biot, M. A., 1965. Mechanics of Incremental Deformations, Wiley, New York.

Budiansky, B., Fleck, N. A., Amazigo, J. C., 1998. On kink-band propagation in fiber composites, Journal of the Mechanics and Physics of Solids 46, 1637-1653.

Fleck, N. A., 1997. Compressive failure of fiber composites, Advances in Applied Mechanics $33,43-117$.

Fleck, N. A., Deng, L., Budiansky, B., 1995. Prediction of kink band width in compressed fiber composites, Transactions of the ASME Journal of Applied Mechanics 62, 329-337.

Golubitsky, M., Schaeffer, D. G., 1984. Singularities and groups in bifurcation theory, Vol. 51 of Applied Mathematical Sciences, Springer-Verlag, New York.

Hobbs, B. E., Means, W. D., Williams, P. F., 1976. An Outline of Structural Geology, Wiley, New York.

Hobbs, R. E., Overington, M. S., Hearle, J. W. S., Banfield, S. J., 1995. Element buckling within ropes and cables, Proceedings of Second European Conference on Flexible Pipes, Umbilicals and Marine Cables, BPP Ltd., pp. 7.1-7.21.

Hunt, G. W., Peletier, M. A., Champneys, A. R., Woods, P. D., Wadee, M. Ahmer, Budd, C. J., Lord, G. J., 1999. Cellular buckling in long structures, Nonlinear Dynamics. To 
appear.

Maynard Smith, J., 1974. Models in Ecology, Cambridge University Press.

Price, N. J., Cosgrove, J. W., 1990. Analysis of Geological Structures, Cambridge University Press.

Reid, S. R., Peng, C., 1997. Dynamic uniaxial crushing of wood, International Journal of Impact Engineering 19, 531-570.

Thompson, J. M. T., Hunt, G. W., 1973. A General Theory of Elastic Stability, Wiley, London.

Zeeman, E. C., 1977. Catastrophe Theory: Selected papers, 1972-1977, Addison-Wesley.

\section{A. Straight Limbs, ShARP Hinges}

An important aspect of the modelling of the preceding sections is the assumption that layers fold in isolated hinges, separated by straight limbs. As remarked in the introduction, this is not obvious from the properties of elastic materials; on the contrary, elastic deformation tends to spread rather than concentrate. The straight-limb, sharp-corner assumption therefore needs justification. The observation that many experiments show such sharp hinges does not reduce this necessity.

Fold creation in natural rock is known to occur under conditions of high pressure. Similarly experiments with analogue materials show that a relatively high confining pressure is necessary for the appearance of kink bands. In this section we shall argue why this confining pressure forces the elastic layers to concentrate the deformation into sharp hinges. The result of the analysis in this section is that for a given hinge angle the hinge region has a finite size $S_{h}=\sigma_{h} \sqrt{E I / P H}$. In this equation $\sigma_{h}$ is a constant that only depends on the hinge angle and is independent of all material parameters. This equation shows that when the thickness $H$ and the elastic properties $E I$ of the layers are kept constant, the hinge size is a decreasing function of the overburden pressure $P$. For high overburden pressures we therefore expect to see the hinges localized into sharp corners, connected by straight limbs.

Before we enter into the modelling details, let us first introduce the underlying concept, as illustrated by Fig. 13. If a stack of incompressible layers is to fold into a similar fold, then by
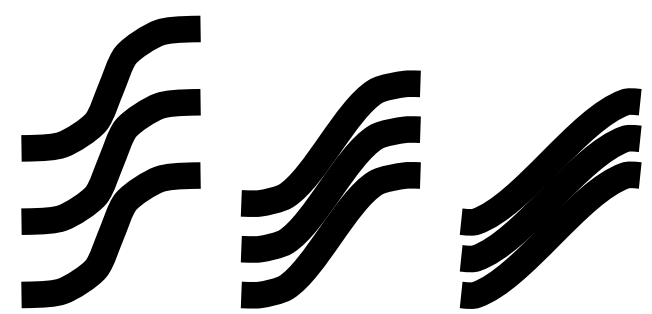

Figure 13: Sharp-angle, straight-limb folds create less voids than rounded folds

simple geometry voids are created at the hinges. These voids are costly, in an energy sense, since they represent work done against the overburden pressure. The amount of void created is very sensitive to the form of the fold, as is shown by Fig. 13. Sinusoid folds create a large amount of void between the layers, while chevron folds are much less costly. This shows from a simple, 
geometric, point of view how a high overburden pressure, which determines the 'unit cost' of void space, can force the layers away from the smooth sinusoid folds into sharp-cornered chevron-type patterns.

The model we derive here is inspired by this observation. Consider a structure consisting of identical thin elastic layers, deformed into a single hinge as in Fig. 14. We suppose that the centerlines of the layers are identical copies of each other, each translated by $(K,-H)$ with respect to the layer above, creating a similar-fold hinge. Choosing $S$ as the arclength coordinate, we represent the centerline by the angle $\theta=\theta(S)$ with the horizontal.

We assume that the layers are long with respect to the hinge zone and we therefore model the fold by unbounded layers, with $\theta \rightarrow 0$ as $S \rightarrow-\infty$ and $S \rightarrow \theta_{\infty}$ as $S \rightarrow \infty$. We also suppose that for large $|S|$ the centerlines are separated by their 'natural' distance $H$; this implies that $\theta_{\infty}=2 \arctan (K / H)$, so that the choice of the hinge angle fixes the value of $K$ and vice versa. Throughout this section we use capitals for dimensional quantities and lower case for their dimensionless equivalents.

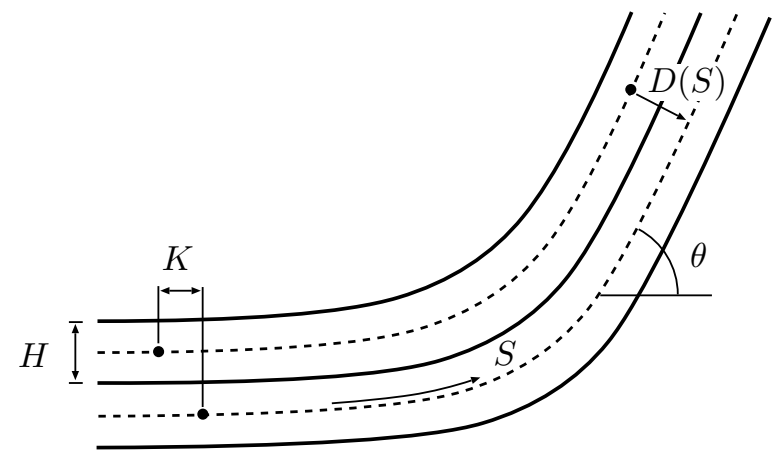

Figure 14: Two layers in a structure folded into a hinge. The centerlines are indicated by dashed lines.

We characterize the optimal configuration as the minimizer of an appropriate energy. With the argument above in mind the two elements that we believe to be important are the bending energy of the individual layers and the penalization of voids owing to the overburden pressure. The bending energy can be modelled by

$$
\frac{E I}{2} \int_{-\infty}^{\infty} \theta^{\prime}(S)^{2} d S
$$

for the penalization of voids we need to make some approximations.

Let $D(S)$ be the distance between the centerline of the layer at the point $S$ and the layer below it (Fig. 14). Writing $(X(S), Y(S))$ for the coordinates of the centerline at $S$, the distance can be characterized as

$$
D(S)^{2}=\min _{T \in \mathbb{R}}|(X(S), Y(S))-(X(T)+K, Y(T)-H)|^{2} .
$$

At the value of $T$ where this minimum is attained, we have

$$
(X(S)-X(T)-K, Y(S)-Y(T)+H) \cdot \frac{d}{d T}(X(T), Y(T))=0 .
$$


Using $X^{\prime}(T)=\cos \theta(T), Y(T)=\sin \theta(T)$, this implies

$$
(X(S)-X(T)-K) \cos \theta(T)+(Y(S)-Y(T)+H) \sin \theta(T)=0 .
$$

Next we make the assumption that $\theta$ varies slowly along the layer, so that $X(S)-X(T) \approx$ $(S-T) \cos \theta(T)$ and $Y(S)-Y(T) \approx(S-T) \sin \theta(T)$. Then (14) becomes

$$
(S-T)-K \cos \theta(T)+H \sin \theta(T) \approx 0 .
$$

We can then write

$$
\begin{aligned}
D(S)^{2}= & (X(S)-X(T)-K)^{2}+(Y(S)-Y(T)+H)^{2} \\
\approx & (X(S)-X(T)-K)(S-T) \cos \theta(T) \\
& \quad+(Y(S)-Y(T)+H)(S-T) \sin \theta(T) \\
& \quad-K(X(S)-X(T)-K)+H(Y(S)-Y(T)+H) \\
\approx & 0-K((S-T) \cos \theta(T)-K)+H((S-T) \sin \theta(T)+H) \\
\approx & K^{2}\left(-\cos ^{2} \theta(T)+1\right)+H^{2}\left(-\sin ^{2} \theta(T)+1\right) \\
& \quad+2 K H \sin \theta(T) \cos \theta(T) \\
= & (K \sin \theta(T)+H \cos \theta(T))^{2} .
\end{aligned}
$$

Since $\theta$ does not vary rapidly along the layer, $\theta(S) \approx \theta(T)$, and we obtain

$$
D(S) \approx|K \sin \theta(S)+H \cos \theta(S)| .
$$

For the solutions that we obtain, $K \sin \theta(S)+H \cos \theta(S)$ is positive, and we shall henceforth drop the absolute value signs.

We penalize the creation of voids by the energy

$$
P \int_{-\infty}^{\infty}(D(S)-H)_{+} d S=P \int_{-\infty}^{\infty}(K \sin \theta(S)+H \cos \theta(S)-H)_{+} d S .
$$

Here $P$ is the overburden pressure, and $x_{+}=\max (x, 0)$. This expression represents the work done into the overburden pressure by the creation of voids between the layers. The optimal configuration is found by minimizing the potential

$$
J=\frac{E I}{2} \int_{-\infty}^{\infty} \theta^{\prime}(S)^{2} d S+P \int_{-\infty}^{\infty}(K \sin \theta(S)+H \cos \theta(S)-H)_{+} d S
$$

over all profiles $\theta$ that tend to 0 at $-\infty$ and to $\theta_{\infty}$ at $+\infty$. Before calculating this minimizer we non-dimensionalize $S$ and $K$ by $H$, i.e. we write $S=H s, K=H k$, and obtain

$$
\begin{aligned}
J & =\frac{E I}{2 H} \int_{-\infty}^{\infty} \theta^{\prime}(s)^{2} d s+P H^{2} \int_{-\infty}^{\infty}(k \sin \theta(s)+\cos \theta(s)-1)_{+} d s \\
& =\frac{E I}{H^{3}}\left\{\frac{1}{2} \int_{-\infty}^{\infty} \theta^{\prime}(s)^{2} d s+\frac{P H^{3}}{E I} \int_{-\infty}^{\infty}(k \sin \theta(s)+\cos \theta(s)-1)_{+} d s .\right\}
\end{aligned}
$$

We define the nondimensional parameter $\lambda^{2}=P H^{3} / E I$. 
A minimizer $\theta$ of $J$ satisfies the Euler equation

$$
-\theta^{\prime \prime}+\lambda^{2}(k \cos \theta-\sin \theta) \mathcal{H}(k \sin \theta+\cos \theta-1)=0,
$$

where $\mathcal{H}$ is the Heaviside function

$$
\mathcal{H}(x)= \begin{cases}1 & \text { if } x>0 \\ 0 & \text { if } x \leq 0\end{cases}
$$

The parameter $\lambda$ can be scaled out of this equation by setting $\sigma=\lambda s$, so that

$$
-\frac{d^{2} \theta}{d \sigma^{2}}+(k \cos \theta-\sin \theta) \mathcal{H}(k \sin \theta+\cos \theta-1)=0 .
$$

The most important result of this modelling can now be obtained even without solving this equation. The fact that (15) is independent of all material parameters implies that for all choices of these parameters the solutions are the same up to a scaling. In terms of $\sigma$, the length of the hinge region is fixed (say $\sigma_{h}$ ); therefore the hinge length of a hinge in laboratory variables is $S_{h}=H s_{h}=\sigma_{h} H / \lambda$. This equation contains the main statement of this section: at constant layer thickness the length of the hinge section is proportional to the ratio $\lambda^{-1}=\sqrt{E I / P H^{3}}$ of bending stiffness and overburden pressure. For instance, doubling the overburden pressure decreases the length of the hinge by a factor $\sqrt{2}$.

It is, however, possible to solve this equation, at least in a partially explicit way. Writing $F(\theta)=(k \sin \theta+\cos \theta-1)_{+}^{2}$ and $f(\theta)=F^{\prime}(\theta)=(k \cos \theta-\sin \theta) \mathcal{H}(k \sin \theta+\cos \theta-1)$, we can multiply (15) by $\theta^{\prime}$ and integrate to obtain

$$
-\frac{1}{2}\left(\frac{d \theta}{d \sigma}\right)^{2}+F(\theta)=c
$$

where $c$ is an integration constant. Since $\theta, \theta^{\prime} \rightarrow 0$ as $\sigma \rightarrow-\infty$, we have $c=0$, and therefore

$$
\frac{d \theta}{d \sigma}=\sqrt{2 F(\theta)}
$$

By inverting the roles of $\theta$ and $\sigma$ we can solve this equation in the form

$$
\sigma=\int_{\arctan k}^{\theta(\sigma)} \frac{d t}{\sqrt{2 F(t)}} .
$$

Solution graphs are plotted in Fig. 15 for different values of $k$. This figure suggests that the length of the hinge zone $-\sigma_{h}$-is independent of $k$ to first approximation. Using the formula

$$
\sigma_{h}=2 \int_{0}^{\arctan k} \frac{d t}{\sqrt{2 F(t)}}
$$

we plot $\sigma_{h}$ as a function of $k$ in Fig. 16. The fact that $\sigma_{h}$ appears to take the value $\pi$ as $k \rightarrow 0$ is no coincidence; indeed if $k$ is small, then $\sin t \sim t, \cos t-1 \sim t^{2} / 2$, and the integral is approximated by

$$
\sqrt{2} \int_{0}^{1} \frac{d \tau}{\sqrt{\tau-\tau^{2} / 2}} .
$$

Here we have substituted $k \tau$ for $t$. This integral has the value $\pi$, as can be verified by substituting $1-\rho$ for $\tau$. 


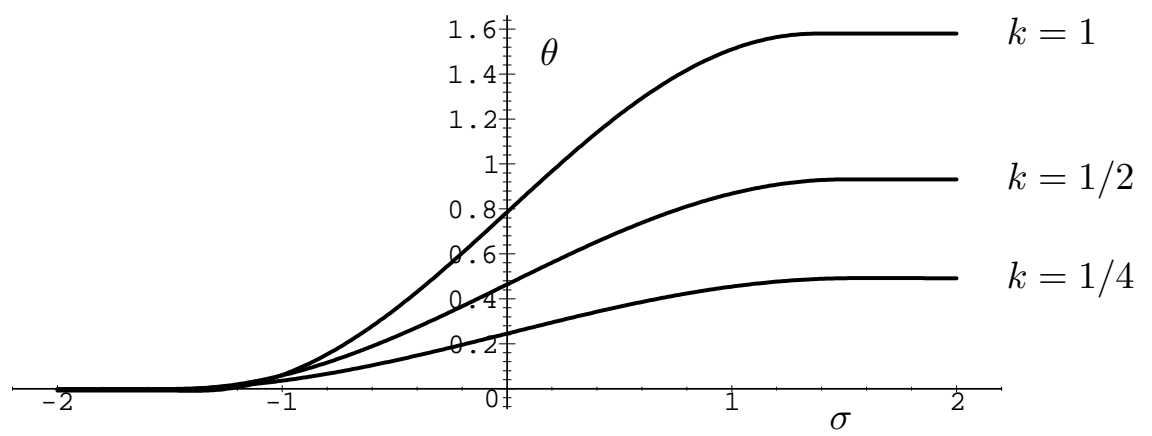

Figure 15: Numerical calculations of the hinge profile. The graph shows the value of $\theta$, the layer angle, as a function of $\sigma$, the non-dimensionalized arc length.

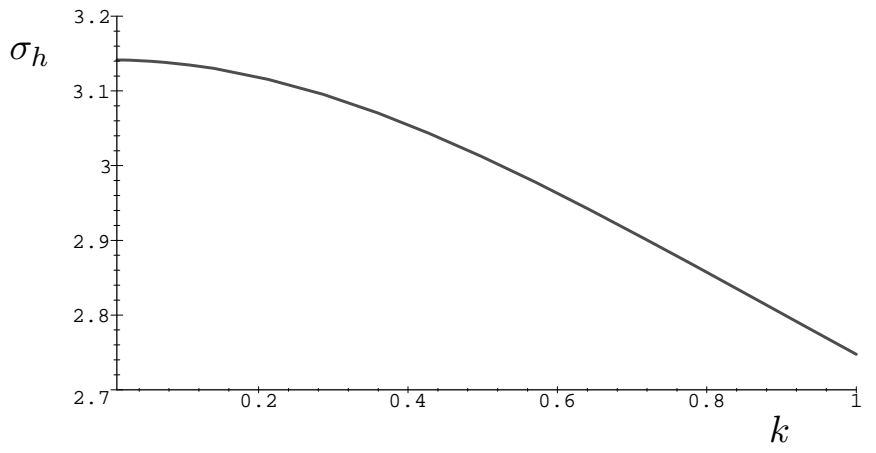

Figure 16: Length of the hinge zone as a function of $k$ 